\title{
Numerical study of inflow equivalence ratio inhomogeneity on oblique detonation formation in hydrogen-air mixtures
}

\author{
Yishen Fang ${ }^{\mathrm{a}}$, Zongmin $\mathrm{Hu}^{\mathrm{a}}$, Honghui Teng ${ }^{\mathrm{b}, \mathrm{a}, *}$, Zonglin Jiang ${ }^{\mathrm{a}}$, Hoi Dick $\mathrm{Ng}^{\mathrm{c}}$ \\ a State Key Laboratory of High Temperature Gas Dynamics, Institute of Mechanics, Chinese Academy of Sciences, Beijing 100190, China \\ ${ }^{\mathrm{b}}$ Department of Mechanics, School of Aerospace Engineering, Beijing Institute of Technology, Beijing 100081, China \\ ${ }^{\mathrm{c}}$ Department of Mechanical, Industrial and Aerospace Engineering, Concordia University, Montreal, QC, H3G 1M8, Canada
}

\section{A R T I C L E I N F O}

\section{Article history:}

Received 28 March 2017

Received in revised form 19 August 2017

Accepted 18 September 2017

Available online 21 September 2017

\section{Keywords:}

Oblique detonation

Equivalence ratio

Mixture inhomogeneity

Detailed chemistry

\begin{abstract}
A B S T R A C T
In this study, numerical simulations using Euler equations with detailed chemistry are performed to investigate the effect of fuel-air composition inhomogeneity on the oblique detonation wave (ODW) initiation in hydrogen-air mixtures. This study aims for a better understanding of oblique detonation wave engine performance under practical operating conditions, among those is the inhomogeneous mixing of fuel and air giving rise to a variation of the equivalence ratio (ER) in the incoming combustible flow. This work focuses primarily on how a variable equivalence ratio in the inflow mixture affects both the formation and characteristic parameters of the oblique detonation wave. In this regard, the present simulation imposes initially a lateral linear distribution of the mixture equivalence ratio within the initiation region. The variation is either from fuel-lean or fuel-rich to the uniform stoichiometric mixture condition above the oblique shock wave. The obtained numerical results illustrate that the reaction surface is distorted in the cases of low mixture equivalence ratio. The so-called "V-shaped" flame is observed but differed from previous results that it is not coupled with any compression or shock wave. Analyzing the temperature and species density evolution also shows that the fuel-lean and fuel-rich inhomogeneity have different effects on the combustion features in the initiation region behind the oblique shock wave. Two characteristic quantities, namely the initiation length and the ODW surface position, are defined to describe quantitatively the effects of mixture equivalence ratio inhomogeneity. The results show that the initiation length is mainly determined by the mixture equivalence ratio in the initiation region. Additional computations are performed by reversing ER distribution, i.e., with the linear variation above the initiation region of uniform stoichiometric condition and results also demonstrate that the ODW position is effectively determined by the ER variation before the ODW, which has in turn only negligible effect on the initiation length.
\end{abstract}

(c) 2017 Elsevier Masson SAS. All rights reserved.

\section{Introduction}

The development of air-breathing hypersonic aircrafts has attracted increasing attention in recent years. The oblique detonation wave (ODW) concept used in detonation-based engines [1] and Ram Accelerators [2] has long been considered as a viable option for achieving the required high efficient propulsion. This kind of aerospace propulsion system inherits the advantages of the Scramjet (Supersonic combustion ramjet), and furthermore achieves the high thermal cycle efficiency through the detonative combustion $[3,4]$. However, it remains challenging experimentally to initiate and subsequently stabilize steady oblique detonations in a high-

\footnotetext{
* Corresponding author.

E-mail address: hhteng@bit.edu.cn (H. Teng).
}

speed flow of combustible mixture, and further systematic theoretical and numerical studies on the oblique detonation initiation and instability need to be performed for advancing the current engineering development of ODW-based propulsion systems.

The ODW phenomenon has been a subject of many theoretical, experimental and lately numerical investigations. Although the basic theoretical foundation for steady ODWs such as wave angles and steady configurations has been well established [5-8], there are still outstanding fundamental problems on the understanding and prediction of the ODW formation and unsteady structures, which are relevant to practical issues for stable operation of the ODW engine. There have been indeed continuous efforts in recent years to perform numerical simulation to describe these phenomena. Since the pioneering work of Li et al. [9] which first described the classical structure of oblique detonation wave composed of a non-reactive oblique shock, an induction region, a set of defla- 
gration waves, and the oblique detonation surface all united at a multi-wave point, other types of initiation structure were also revealed from more recent numerical studies. These include the smooth transition from a curved oblique shock to ODW [10-12] and several more complex ODW formation of different wave configurations, for example, with the induction region observed to be ended by an internal Chapman-Jouguet (CJ) detonation wave for low inflow Mach number condition rather than a set of deflagration waves in the classical ODW structure [13-17].

Besides the complex formation structures, recent high resolution numerical simulations have also demonstrated clearly the inherent instability of the ODW. The fine scale, instability features on the oblique detonation surfaces with sets of transverse waves are typical of an unstable frontal structure of normal cellular detonations [18-22]. Furthermore, multi-mode detonation engines which combine the potential advantage of both ODW and the pulsed normal detonation $[23,24]$ are proposed, and the ODW induced by a confined wedge is also analyzed numerically and theoretically $[25,26]$.

For the application of oblique detonations for propulsion, their flow structures and locations need to be predicted to facilitate the engineering design and to control the engine performance. However, in practical situations the incoming reactive flow will unavoidably consist of various types of flow inhomogeneity. Hence, it is crucial to understand the oblique detonation structures with inflow perturbations [27-29]. In normal detonations, the initiation features and their correlation with other dynamic parameters such as cell width have been studied widely [30-33], but only few equivalent studies are performed on the ODW formation especially including inflow disturbance such as non-uniformity in the mixture equivalence ratio denoted in this paper by "ER". Sislian et al. [34] described the effects of incomplete fuel/air mixing on two types of ramjets performance characteristics by assuming a Gaussian distribution of equivalence ratio in the combustible mixture flow, and the deflagration distortion is observed clearly. Zhang et al. [35] studied the formation of ODW with various ER and found that the initiation length as function of ER displays a classical "V-shaped" curve, similar to the relation between detonation cell size and initiation energy [36]. Iwata et al. [37] simulated the shock-induced combustion from a supersonic spherical projectile, illustrating several shock-flame configurations induced by inflow ER inhomogeneity. They also performed simulations on wedge-stabilized oblique detonations [38] with different Gaussian ER distributions, demonstrating that the near-wedge deflagration fronts are distorted into the complicated surface, generating the so-called "V-shaped" deflagration front and "V+Y" Mach stem.

Based on the previous studies on how the ER inhomogeneity influences ODW structures [35,37], the emphasis of this work is on how such inhomogeneity in the initiation region changes the characteristic parameters, such as the initiation length and ODW position, under practical operating conditions of ODW engines. Our previous study [39] demonstrates that considering the high flight altitude of ODW engines, the transition from OSW (oblique shock wave) to ODW is achieved by a curved shock, different from the abrupt transition structure studied widely [9]. This numerical study was performed using an ideal incoming well-premixed, combustible mixture with uniform ER. As part of our continuous effort to study the fundamental problem of ODW formation under practical flight conditions, the present numerical investigation addresses the effects of inflow ER inhomogeneity with similar flight characteristics. First, three cases with uniform ER values are simulated and a numerical mesh resolution study is performed. A lateral linear distribution of the mixture equivalence ratio within the initiation region is then introduced in the simulation and adjusted in several test cases, generating a variable fuel-lean or fuel-rich mixture in the initiation region before the oblique shock wave.

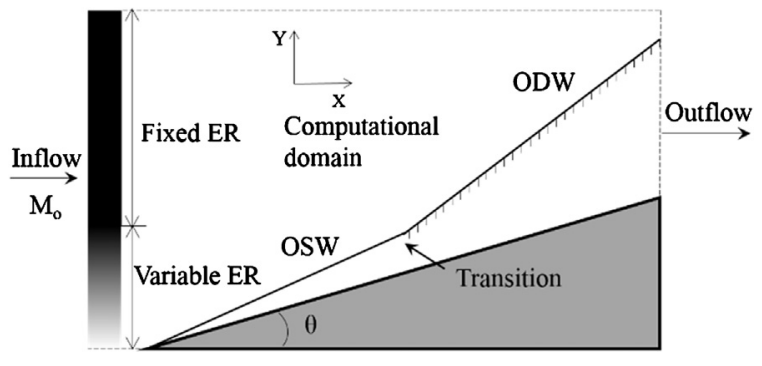

Fig. 1. Schematic of a typical oblique detonation wave.

Analysis is performed on the simulation results by defining two characteristic length scales, namely, the initiation length and the ODW surface position, to assess the effects of ER inhomogeneity.

\section{Physical and numerical models}

A simple schematic of the oblique detonation wave induced by a semi-finite wedge from an inflowing combustible gas mixture is shown in Fig. 1. A supersonic combustible gas mixture with an incident Mach number $M_{o}$ reflects on the two-dimensional wedge and generates first an OSW. The OSW will trigger combustion depending on $M_{O}$ and incoming flow pressure and temperature (i.e., enthalpy), and under appropriate conditions will initiate the oblique detonation formation. Previous results [40] showed that the viscosity and boundary layer have little effects on this structure except changing the boundary layer thickness slightly, so most of the successive results use the inviscid calculation, e.g. [11-22]. Following this assumption, the governing equations are simplified as two-dimensional multi-species Euler equations and can be written as follows:

$\frac{\partial \mathbf{U}}{\partial t}+\frac{\partial \mathbf{E}}{\partial x}+\frac{\partial \mathbf{F}}{\partial y}=\mathbf{S}$

where:

$\mathbf{U}=\left\{\begin{array}{c}\rho_{1} \\ \vdots \\ \rho_{n} \\ \rho u \\ \rho v \\ e\end{array}\right\}, \quad \mathbf{E}=\left\{\begin{array}{c}\rho_{1} u \\ \vdots \\ \rho_{n} u \\ \rho u^{2}+p \\ \rho u v \\ (e+p) u\end{array}\right\}$

$\mathbf{F}=\left\{\begin{array}{c}\rho_{1} v \\ \vdots \\ \rho_{n} v \\ \rho u v \\ \rho v^{2}+p \\ (e+p) v\end{array}\right\}, \quad \mathbf{S}=\left\{\begin{array}{c}\dot{\omega}_{1} \\ \vdots \\ \dot{\omega}_{n} \\ 0 \\ 0 \\ 0\end{array}\right\}$.

In the above equations $\rho_{i}(i=1 \ldots n)$ is the $i$-th species density and the total density is $\rho=\sum_{i=1}^{n} \rho_{i}$. u and $v$ are the velocity in the $x$ - and $y$-direction. Total specific energy $e$ is calculated as

$e=\rho h-p+\frac{1}{2} \rho\left(u^{2}+v^{2}\right)$

where the specific enthalpy can be written as $h=\sum_{i=1}^{n} \rho_{i} h_{i} / \rho$ and the species specific enthalpy $h_{i}$ can be obtained from the 9 -coefficient NASA polynomial representation [41]. Equation of state is

$p=\sum_{i=1}^{n} \rho_{i} R_{i} T$ 
where $R_{i}$ is the $i$-th species gas constant and $T$ is the gas temperature. $\dot{\omega}_{i}$ is the species specific mass production rate, which is determined from the chemical reaction model.

Governing equations are solved numerically on adaptive unstructured quadrilateral grids [42] with the MUSCL-Hancock scheme [43], which achieves a second-order accuracy in space and time by re-constructing the Riemann problem solved using the HLLC approximate Riemann solver on the intercell boundary. A hydrogen-air chemical reaction mode which includes 11 species $\left(\mathrm{H}_{2}, \mathrm{O}_{2}, \mathrm{O}, \mathrm{H}, \mathrm{OH}, \mathrm{HO}_{2}, \mathrm{H}_{2} \mathrm{O}_{2}, \mathrm{H}_{2} \mathrm{O}, \mathrm{N}_{2}, \mathrm{~N}, \mathrm{NO}\right)$ and 23 chemical reactions, is selected from the widely used CHEMKIN package [44]. The DVODE package [45] is used to solve the stiff system of equations generated by the chemical kinetics. The slip wall reflecting boundary condition is used on the wedge surface, and the other boundaries are interpolated under the assumption of the zero firstorder derivatives of all flow parameters.

This numerical methodology has been used previously to investigate the ODW with homogeneous inflow [35,39], i.e., fixed ER. In this study, the inflow ER inhomogeneity is introduced by assuming a lateral linear distribution. It is noted that to mimic the ER variation in realistic scenarios, previous studies [27,28,34,38] generally use either Gaussian or sinusoidal distributions, covering the whole inflow regime of the computational domain. To isolate its effect on the initiation and further simplify the analysis, this study considers only the inhomogeneity before the OSW, which can be viewed as the initiation region. In this way the effect of ER inhomogeneity on the ODW surface is eliminated, facilitating the quantitative analysis of the inhomogeneous fuel-air mixture on the initiation process. In addition, the lateral ER variation in the incoming flow within the initiation region is assumed to be linear for an ease of analysis. This represents the simplest scenario to exclude the ER nonlinearity effects although the Gaussian distribution is usually more representative of a realistic situation $[27,28,38]$. A representation of the inflow ER inhomogeneity is also displayed in Fig. 1. The portion with fixed ER before the ODW is set to be stoichiometry without specific description, i.e., $\mathrm{H}_{2}: \mathrm{O}_{2}: \mathrm{N}_{2}=2: 1: 3.76$. In the variable ER region, mainly before the OSW, the pressure and temperature are the same for all cases, and ER is adjusted by changing at the bottom of left boundary the ratio of $\mathrm{H}_{2}$, e.g., $\mathrm{H}_{2}: \mathrm{O}_{2}: \mathrm{N}_{2}=$ 4:1:3.76 corresponding to $\mathrm{ER}_{\mathrm{b}}=2.0$ and $\mathrm{H}_{2}: \mathrm{O}_{2}: \mathrm{N}_{2}=0: 1: 3.76$ for $\mathrm{ER}_{\mathrm{b}}=0.0$ with inert gases only without any $\mathrm{H}_{2}$ fuel at the wedge tip. The upper boundary value in the variable ER region is the same as the fixed ER, with default value 1.0, so the bifurcation parameter is the lower boundary $\mathrm{ER}_{\mathrm{b}}$ of various test cases, varying from 0.0 to 2.0 in this study (where the subscript "b" stands for the value at the bottom boundary at the wedge tip). After prescribing the lower boundary $E_{b}$, the linear interpolation gives the ER distribution as a function of the lateral position to model the inflow ER inhomogeneity. In short, using this simplest variational form for the fuel-air composition allows the present study to vary one single parameter $E R_{b}$ to investigate different degree of ER variation of the inflow combustible mixture and focus primarily on how the key characteristic features (lengths) responds to such non-uniformity.

\section{Results and discussion}

\subsection{Basic ODW structure and resolution study}

The model of ODW engines proposed by Dudebout et al. [46] and used in the later research $[47,48]$ assumes that an inflow is compressed twice by weak oblique shock waves before the detonation initiation chamber. This study follows this model and assumes the flight conditions with an altitude of $25 \mathrm{~km}$, aircraft flight Mach number $M_{\text {flight }}=10$ and the deflection angle $\theta=12.5^{\circ}$. The latter refers to the inflow turning angle induced by the fore body compression, which is used in the following initial condition calcula-
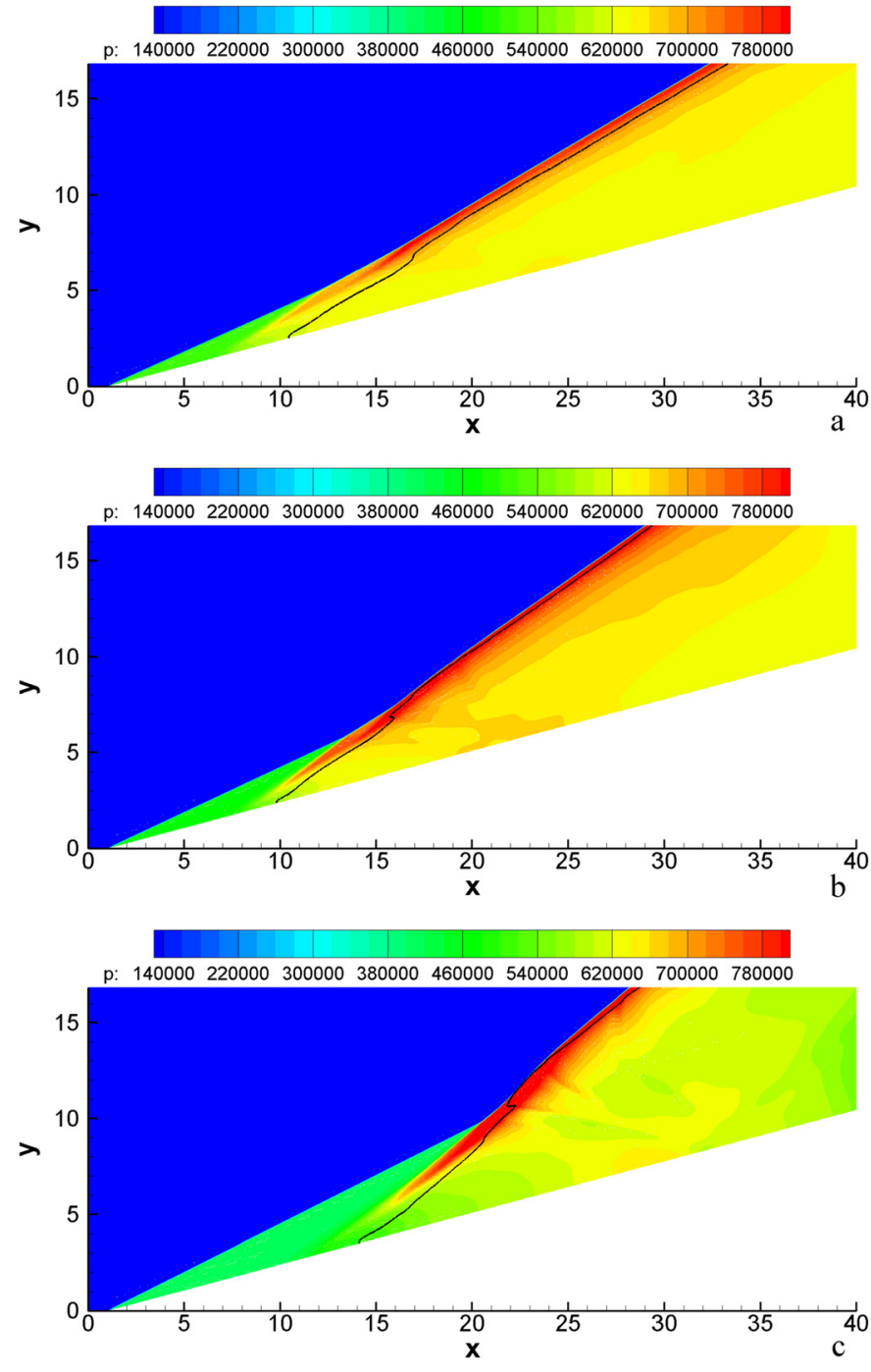

Fig. 2. Pressure fields of oblique detonation structures with $E R=0.5$ (a), 1.0 (b) and 2.0 (c).

tion. Based on the implicit relation between the oblique shock and deflection angles, the oblique shock angle can be calculated and then used to compute the post-OSW pressure and temperature. The flight conditions considered here result in the static pressure of $119 \mathrm{KPa}$ and static temperature of $998 \mathrm{~K}$ with corresponding $M_{0}=4.3$. Nevertheless, the ER variations induce different inflow species fractions and sound speeds, so it is impossible to keep both the velocity and the Mach number $M_{0}$ the same. Considering the characteristics of hypersonic engines, fixing the inflow velocity rather than the Mach number appears to be an option for the present analysis. Hence, the inflow velocity of $3205 \mathrm{~m} / \mathrm{s}$ is adopted in the following simulation cases, corresponding to $M_{0}=4.3$ in the case of $\mathrm{ER}=1.0$. The computational domain is $40 \mathrm{~mm} \times 20 \mathrm{~mm}$, with the default finest grid scale of $0.025 \mathrm{~mm}$. The wedge starts from $x=1 \mathrm{~mm}$, whose angle $\theta$ is fixed to be $15^{\circ}$ in all cases. For simplicity, the unit for all length dimensions is in $\mathrm{mm}$ and so omitted in the figures.

First, three cases with fixed ER are simulated with the grid size of $0.025 \mathrm{~mm}$, and results are shown in Fig. 2. The black line in Fig. 2 denotes the end of the induction zone, corresponding to the temperature of $2200 \mathrm{~K}$ in this study. For the same combustible mixture with $\mathrm{ER}=1.0$, the steady Zel'dovich-von Neumann-Döring (ZND) structure of the corresponding CJ detonation can be computed using the CHEMKIN package [44]. This 

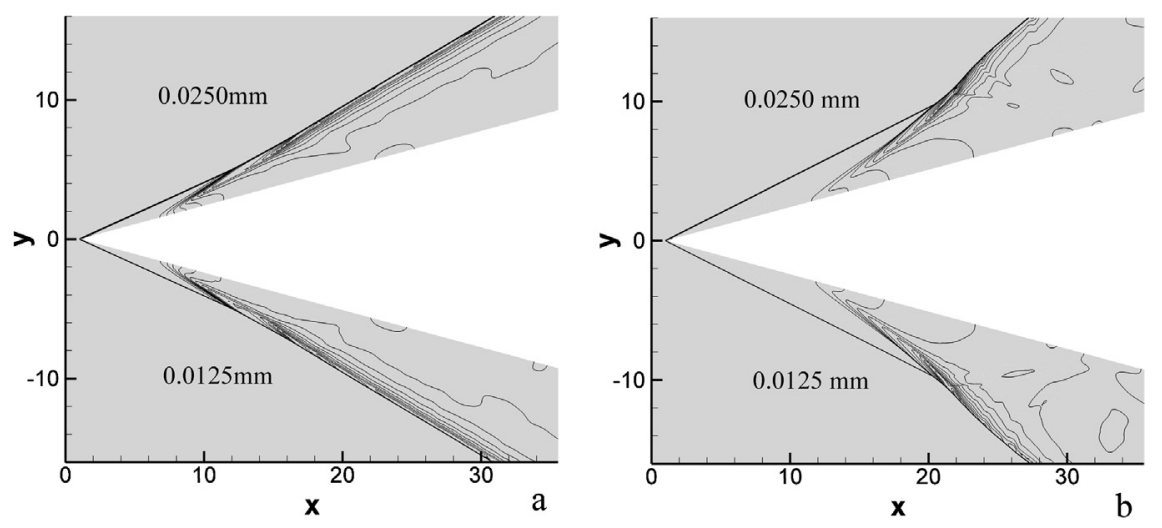

Fig. 3. Pressure fields of ODW with different grid scales of ER $=0.5$ (a) and 2.0 (b).
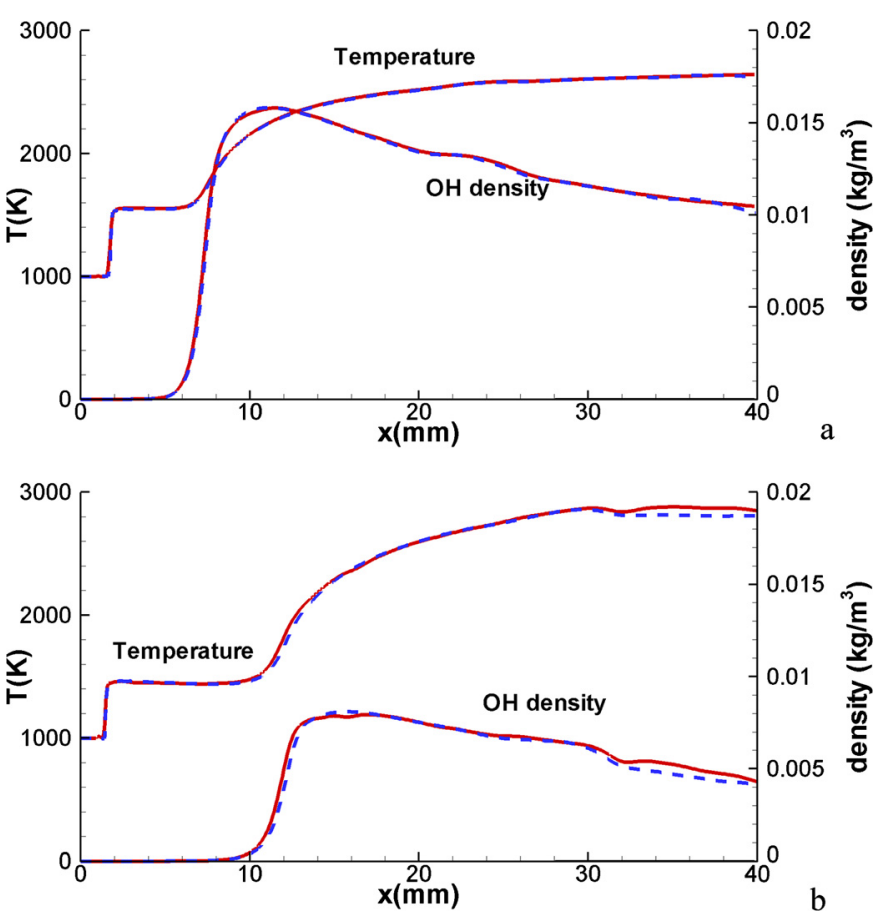

Fig. 4. Temperature and $\mathrm{OH}$ density plots along the wedge for two $\mathrm{ER}=0.5$ (a) and 2.0 (b) with two different grid scales (solid line for $0.025 \mathrm{~mm}$ and dotted line for $0.0125 \mathrm{~mm}$ ). (For interpretation of the references to color in this figure legend, the reader is referred to the web version of this article.)

temperature $2200 \mathrm{~K}$ corresponds to the end of the ZND induction zone, i.e., the inflection point of the temperature profile of $\mathrm{CJ}$ detonation, and it is chosen to identify the reaction surface in this study. It should be noted that oblique detonations are overdriven with different overdriven degrees and hence, this definition only provides a rough representation of the reaction surface. Nevertheless, it gives a quantitative idea on the reactive flow characteristics. Numerical results show that the formation of ODW is developed from the OSW, and both smooth and abrupt transition can be observed, similar to previous studies. Furthermore, the case of ER = 1.0 has the smallest induction zone length. In other words, the induction zone length increases either by increasing or decreasing the overall mixture ER. This agrees with the previous study [35] and the effect is attributed to the increase in ignition delay time when the global mixture ER deviates away from stoichiometric condition ER $=1.0$.

To verify the convergence of the numerical results, Fig. 3 compares the pressure fields of two cases with different grid resolutions. In each frame of Fig. 3 , the upper portion shows simulation results with the grid of $0.0250 \mathrm{~mm}$, and the lower portion shows the results with half the original chosen grid size (i.e., $0.0125 \mathrm{~mm}$ ). In both cases, the difference in the overall wave structure is almost negligible, except the slight discrepancy of contour positions in the combustion product. For better illustration, the corresponding temperature and $\mathrm{OH}$ density plots of the two ER cases along the wedge are also given in Fig. 4 showing again good agreement between the results from the two grid resolutions. Therefore, a resolution of $0.025 \mathrm{~mm}$ is considered sufficient to study the present problem and is selected as the finest grid size. For reference, using the grid size of $0.025 \mathrm{~mm}$ yields $10-20$ grid points per half-reaction zone length along the streamline behind the ODW. The same or less effective grid resolution was also used in recent studies [35,39] which also show that both the structure and instability of oblique detonation surfaces can be correctly captured with this resolution.

\subsection{Effect of equivalence ratio on the ODW formation structure}

With the fuel-lean inflow before the OSW, the flow fields displayed by pressure contours and temperature contour (black) line of $2200 \mathrm{~K}$ are shown in Fig. 5. The ODW initiation is achieved by the smooth transition from the curved OSW, like the cases of fixed $\mathrm{ER}=0.5$ and 1.0 shown in Fig. 2. With the lowest $\mathrm{ER}_{\mathrm{b}}=0.1$, the temperature on the wedge will not attain $2200 \mathrm{~K}$, so the front tip of the reaction surface locates above the wedge. By increasing the $\mathrm{ER}_{\mathrm{b}}$ to 0.2 or 0.3 , a portion of the reaction surface remains above the wedge, but the position of the reaction front tip starts to move gradually upstream. With $\mathrm{ER}_{\mathrm{b}}=0.4$, the front tip moves upstream further and locates on the wedge. Because the equivalence ratio of the combustible mixture flowing into the oblique detonation surface is fixed to 1.0, the ODW wave angles remain therefore the same in all cases. Compared with the homogeneous case with uniform $\mathrm{ER}=1$ throughout the lateral direction shown in Fig. $2 \mathrm{~b}$, these structures display the complicated reaction surface in the initiation region, mainly denoted by the temperature contour. Due to the lack of fuel, the heat release near the wedge is delayed, generating the distorted reaction surface. This is similar to the recent study by Iwata et al. [38] which elucidates the distorted deflagration featured by a convex "V-shaped" flame toward upstream.

To clarify the features of ODW with inflow ER inhomogeneity, the extreme case of $\mathrm{ER}_{\mathrm{b}}=0.0$, i.e., $\mathrm{H}_{2}: \mathrm{O}_{2}: \mathrm{N}_{2}=0: 1: 3.76$ on the wedge, is simulated and shown in Fig. 6 . It can be observed that the density is high but temperature is low near the wedge, as illustrated in Fig. 6a and 6b. The low temperature derives from the lack of combustible fuel and in conjunction with the near uniform pressure in the combustion products (Fig. 5), result in high mixture density. Furthermore, the mixture density near the wedge is mainly the gas species of $\mathrm{N}_{2}$ and $\mathrm{O}_{2}$ with less $\mathrm{H}_{2}$ (see Fig. 6c). The species $\mathrm{OH}$ density demonstrates that the reaction is intense behind the ODW but weak near the wedge due to the absence 

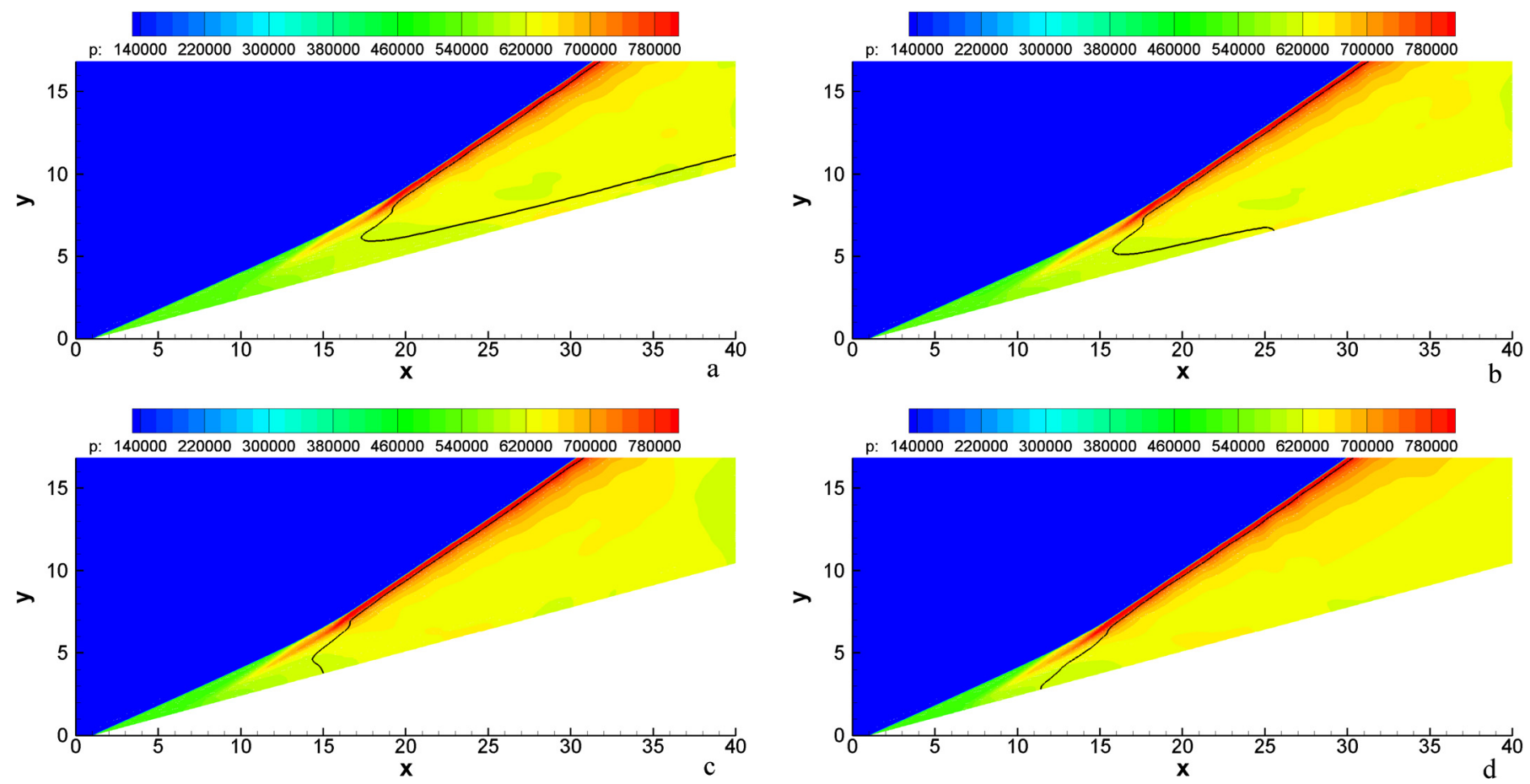

Fig. 5. Pressure fields of oblique detonation structures with the lower $\mathrm{ER}_{\mathrm{b}}=0.1$ (a), 0.2 (b), 0.3 (c), and 0.4 (d).
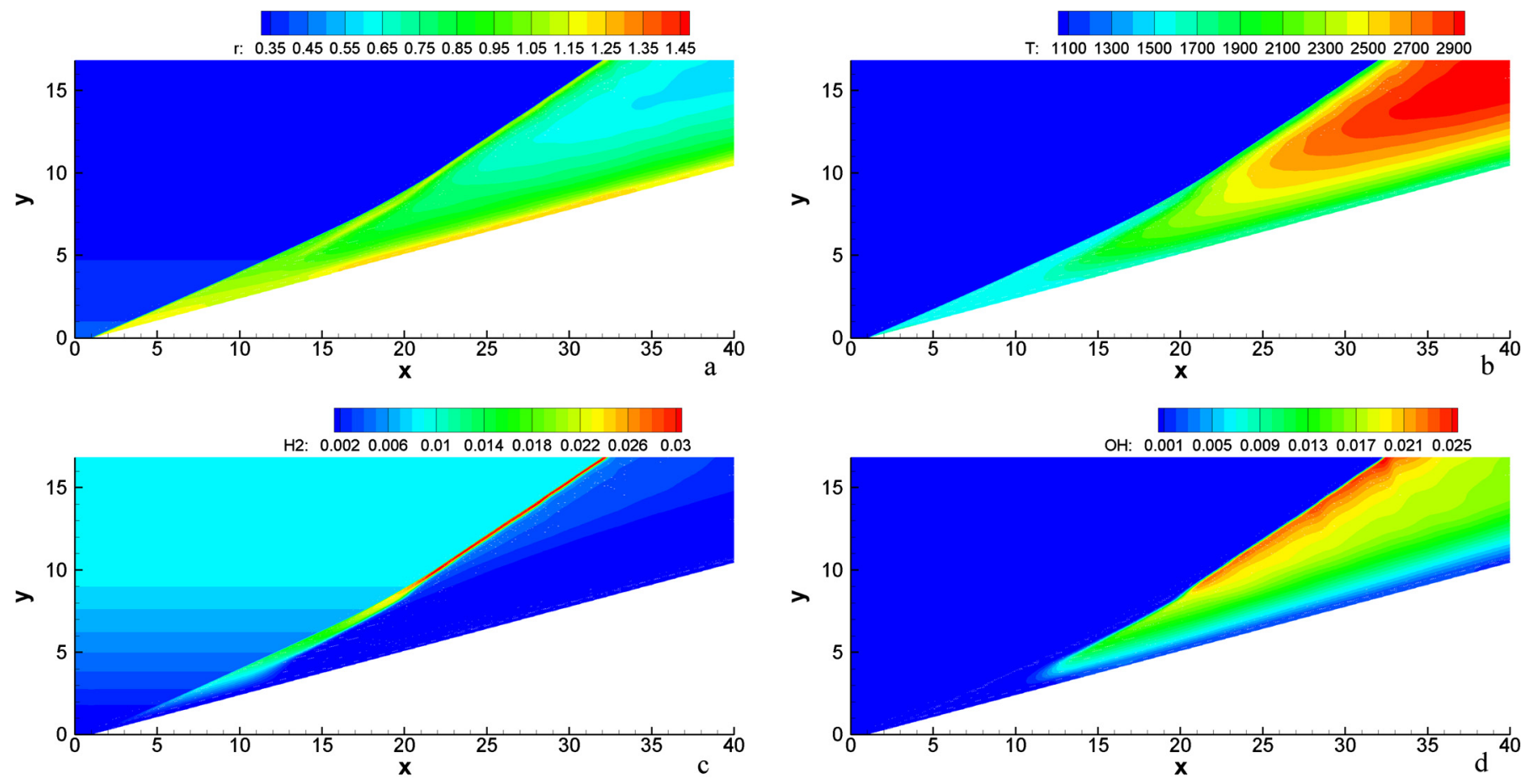

Fig. 6. Total mixture density (a), temperature (b), $\mathrm{H}_{2}$ density (c) and $\mathrm{OH}$ density (d) of oblique detonation structures with $\mathrm{ER}_{\mathrm{b}}=0.0$.

of $\mathrm{OH}$. Generally this structure is influenced by the inflow ER inhomogeneity, showing some special features different from those in the well-premixed uniform inflow. However, with the parameters simulating the ODW engines in high flight conditions in this study, very irregular deflagration waves coupled with compression or shock waves [38] are not observed, demonstrating effects of inflow ER inhomogeneity are not pronounced in the practical scenario considered in this study.

Further cases with a wider $\mathrm{ER}_{\mathrm{b}}$ variation are shown in Fig. 7. It can be observed that the leading front tip of the reaction sur- face moves downstream when ER increasing from 0.8 to 2.0. At the same time, the OSW and ODW angles increase, and the transition type changes from the smooth one to the abrupt one. The minimum induction zone length appears for an $\mathrm{ER}_{\mathrm{b}}$ of 0.8 , which is the same as that in the study of the homogeneous inflow [35]. The increase of ODW angles when $\mathrm{ER}_{\mathrm{b}}$ increases stems from the change of the inflow Mach number. By keeping the inflow velocity the same, the corresponding Mach number $M_{o}$ actually decreases when ER increases due to a higher amount of hydrogen present in the mixture. It deserves to be mentioned that the distorted 

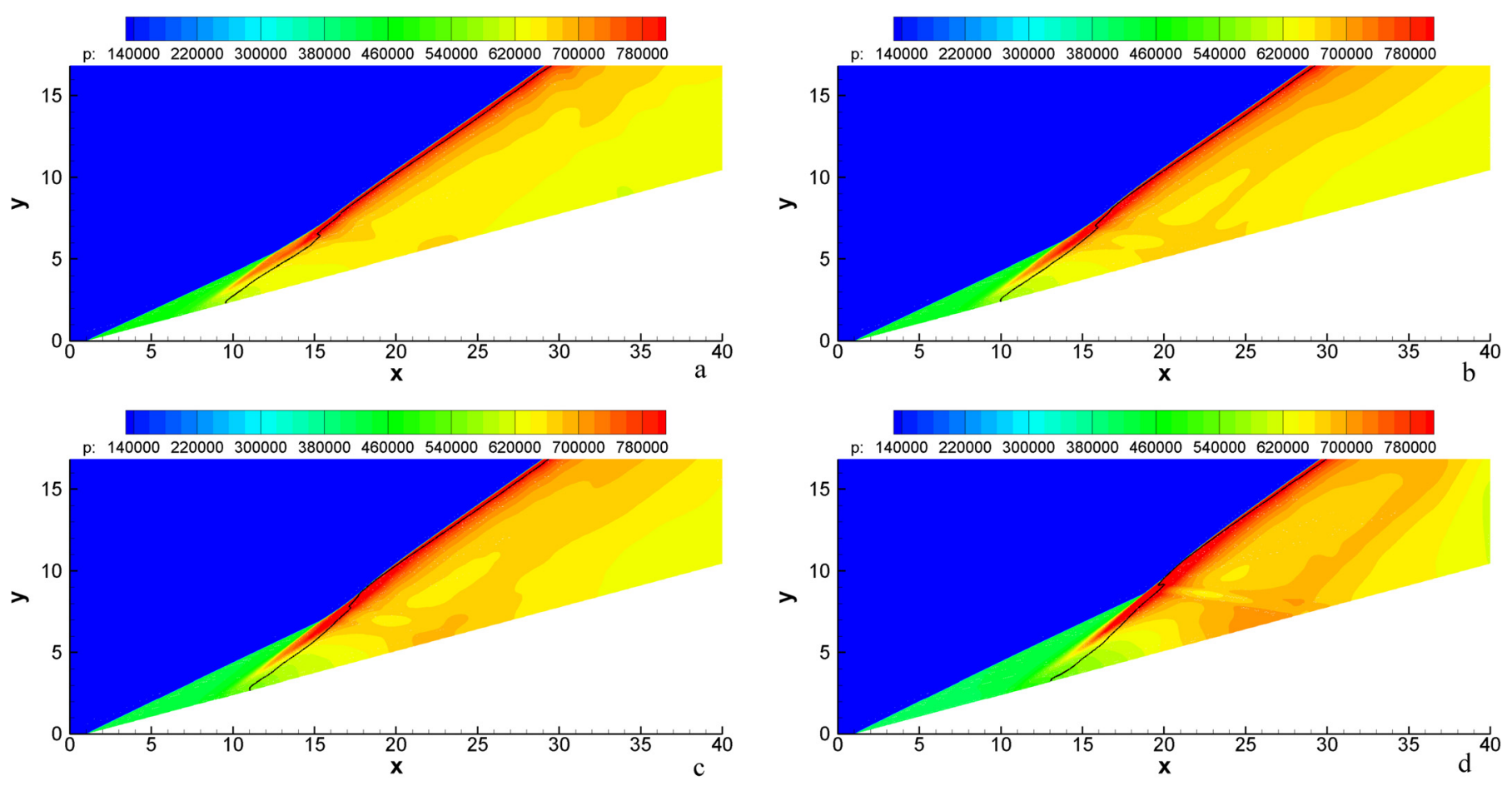

Fig. 7. Pressure of oblique detonation structures with $E_{\mathbf{b}}=0.8$ (a), 1.2 (b), 1.6 (c), and 2.0 (d).
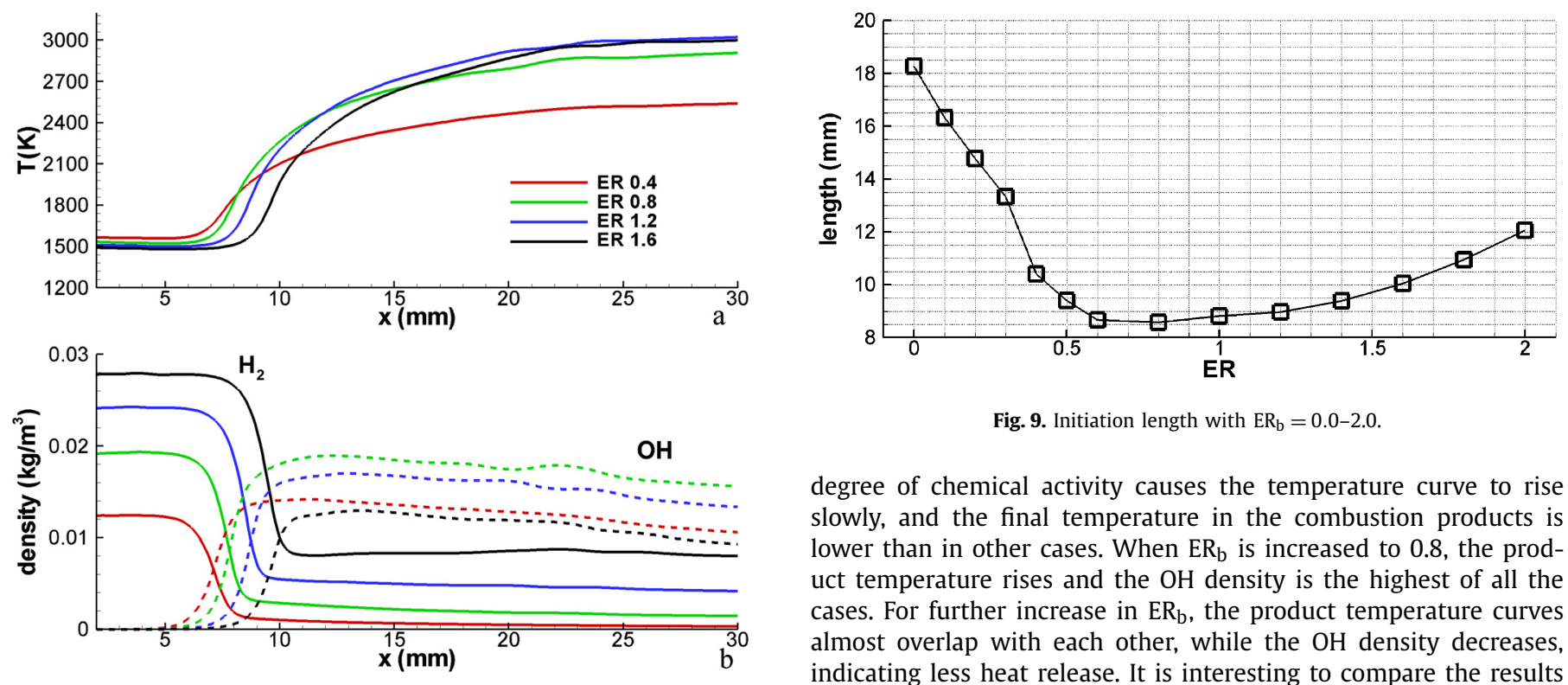

Fig. 9. Initiation length with $\mathrm{ER}_{\mathrm{b}}=0.0-2.0$.

degree of chemical activity causes the temperature curve to rise slowly, and the final temperature in the combustion products is lower than in other cases. When $\mathrm{ER}_{\mathrm{b}}$ is increased to 0.8 , the product temperature rises and the $\mathrm{OH}$ density is the highest of all the cases. For further increase in $\mathrm{ER}_{\mathrm{b}}$, the product temperature curves almost overlap with each other, while the $\mathrm{OH}$ density decreases, indicating less heat release. It is interesting to compare the results of the ER 0.4 and 1.6 cases. The $\mathrm{OH}$ density curves in the product are close to each other, but the temperature curves deviate, demonstrating the fuel lean and fuel rich inhomogeneity has different combustion features.

"V-shaped" flame is not observed in the fuel-rich cases. Theoretically, by further increasing $\mathrm{ER}_{\mathrm{b}}$, the fuel rich inflow near the wedge could induce a similar wave configuration as for the fuel-lean inflow. Nevertheless, the extremely high $\mathrm{ER}_{\mathrm{b}}$ cases, such as ER above 4.0 , were not of practical interest in this study.

To describe the chemistry in the initiation region with the composition inhomogeneity, the temperature and species density on the wedge for the cases from $\mathrm{ER}_{\mathrm{b}} 0.4$ to 1.6 are plotted in Fig. 8 . The post-oblique shock temperature, denoted by the plateau in Fig. 8a (approximately at $x=0-7 \mathrm{~mm}$ ), decreases with increasing $\mathrm{ER}_{\mathrm{b}}$. Overall, it may appear that the ignition occurs first with $\mathrm{ER}_{\mathrm{b}}=0.4$ due to a higher post-oblique shock temperature. However, for fuel-lean condition with the lowest $\mathrm{ER}_{\mathrm{b}}=0.4$, the lower

\subsection{Discussion on quantitative features}

For a quantitative assessment of the effect of ER inhomogeneity on the ODW formation, the initiation lengths and ODW surface positions along $y=20$ are shown in Fig. 9 and Fig. 10, respectively. The initiation length is defined as the distance in the $x$-direction between the front of the wedge and the upstream tip of the reaction surfaces as indicated by the $2200 \mathrm{~K}$ temperature contour line. As shown in Fig. 9, the initiation length decreases when $E_{b}$ is increased from 0.0 until 0.8. In the cases with $E_{b} 0.0$ to 0.3 , the length decreases almost linearly; but such decrease becomes modest in the cases with $\mathrm{ER}_{\mathrm{b}} 0.4$ to 0.8. Reversely, a modest increase 


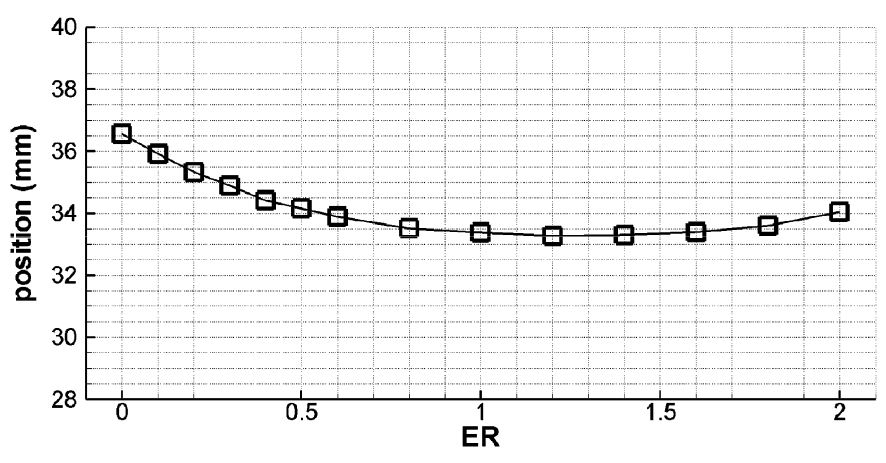

Fig. 10. Oblique detonation wave surface position along $y=20$ with $\mathrm{ER}_{\mathrm{b}}=0.0-2.0$.
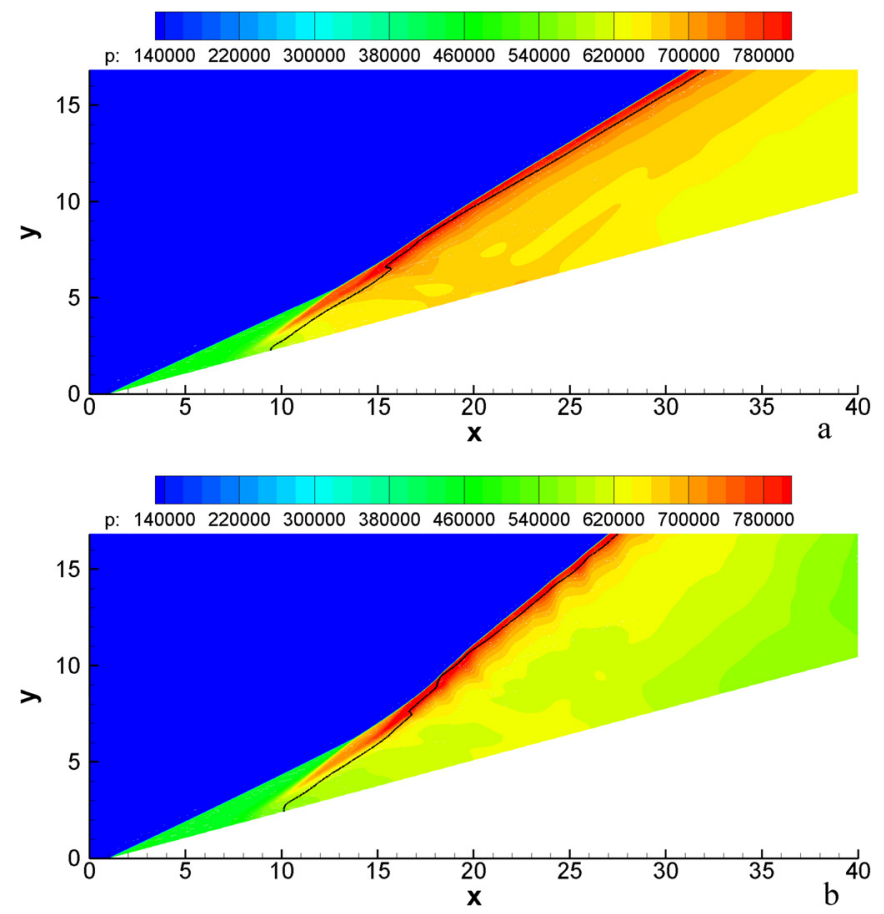

Fig. 11. Pressure fields of oblique detonation structures with upper $E R=0.5$ (a) and 2.0 (b).

can be observed when ER changes from 0.8 to 2.0. The induction length for $\mathrm{ER}_{\mathrm{b}}=2.0$ corresponds roughly to the length found with $\mathrm{ER}_{\mathrm{b}}$ between 0.3 and 0.4 .

The ODW position is another key quantitative parameter for the engine design, but still lacks a universal definition and discussion. Although it is strongly coupled with the initiation length, the ODW position provides another quantity for the engine designer to control and assess if an ODW can be established within the physical constraints. In this study, we define it by the ODW surface position along the line $y=20$, and the results are shown in Fig. 10. Compared with the initiation length, the ODW position is not significantly influenced by the inflow ER inhomogeneity. By increasing $\mathrm{ER}_{\mathrm{b}}$ from 0.0 , the ODW position moves upstream slowly at first, but soon becomes insensitive to the $\mathrm{ER}_{\mathrm{b}}$ variation above $\mathrm{ER}_{\mathrm{b}}=0.8$. The ODW surface position dependence on the ER can be interpreted by analyzing the flow fields. When $\mathrm{ER}_{\mathrm{b}}$ is increased above 0.8 , the initiation length increases while the oblique detonation angle is found to decrease. The former moves the surface position downstream while the later tends to adjust the surface position upstream. These two competing results thus makes the ODW position insensitive to the $\mathrm{ER}_{\mathrm{b}}$ variation as shown in Fig. 10.

To further investigate the effects of inflow ER inhomogeneity on the ODW formation, additional computations are performed with

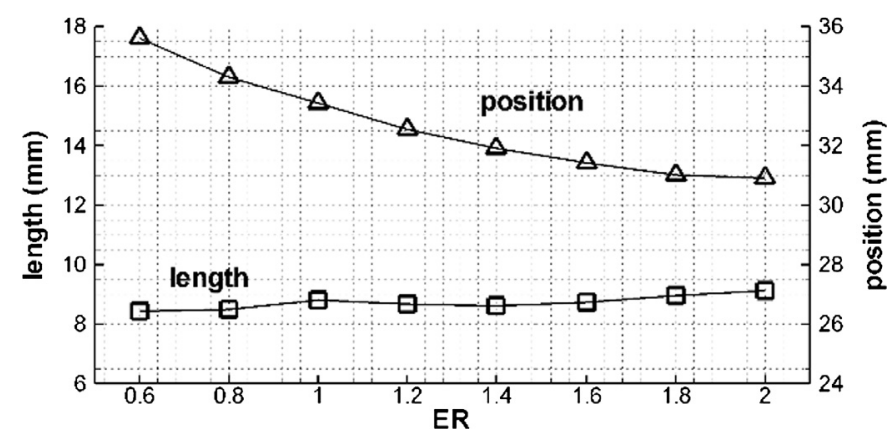

Fig. 12. Initiation length and ODW surface position along $y=20$ with upper ER $=$ $0.6-2.0$.

the reversed ER distribution. So far, simulations are performed with the introduction of various linear ER distributions before the OSW in the initiation region, while keeping the ODW surface region with uniform ER 1.0. These following computations vary the upper $\mathrm{ER}_{\mathrm{u}}$ before the ODW from 0.6 to 2.0, but keep the lower ER before the OSW fixed to 1.0. Two typical flow fields, with the $\mathrm{ER}_{\mathrm{u}}=0.5$ and 2.0 before the ODW, are shown in Fig. 11. It can be observed that the initiation lengths are rather close, but the oblique detonation angles differ significantly, as a result the ODW surface positions deviate significant from each other. The parametric results of the initiation length and ODW position are shown in Fig. 12. Generally the initiation length is almost independent on $E_{\mathrm{u}}$ and hence the upper ER variation, but the ODW surface position is sensitive and decreases when $\mathrm{ER}_{\mathrm{u}}$ increases. Compared with the results given in Figs. 9 and 10, the initiation length is mainly determined by $\mathrm{ER}_{\mathrm{b}}$ before the OSW, but the ODW position is significantly influenced by the $\mathrm{ER}_{\mathrm{u}}$ before the ODW with changes in the oblique detonation angle.

\section{Concluding remarks}

Numerical simulations of oblique detonation wave initiation in hydrogen-air mixtures are performed to investigate the effect of inflow mixture ER inhomogeneity typically resulted from different degree of fuel-air mixing in practical ODW engines. The inflow ER inhomogeneity is introduced by assuming a lateral linear ER distribution before the OSW and the $\mathrm{ER}_{\mathrm{b}}$ at the lower boundary is used as the modeling parameter and to control the steepness of the ER variation in the initiation region. Numerical results demonstrate that the reaction surface is distorted in the cases of low $\mathrm{ER}_{\mathrm{b}}$, resulting into the so-called "V-shape" deflagration. However, with the practical initial condition and flow parameters considered in this study, effects of the inflow ER inhomogeneity is weakened and the very irregular deflagration waves, coupled with compression or shock waves, do not appear. The temperature and species density with $\mathrm{ER}_{\mathrm{b}}=0.4$ to 1.6 also demonstrate that the fuel-lean and fuel-rich inhomogeneity have different effects on the combustion features.

Different from previous works in the literature, this study takes on a simplified ER inhomogeneity, i.e., a linear variation rather than the generally used Gaussian distribution. It deserves to mention that the linear distribution is only an ideal consideration, not as realistic as the Gaussian distribution. However, it eliminates the nonlinear effects of Gaussian distribution, benefiting to the quantitative analysis and comparison. To assess the effect of ER inhomogeneity, two characteristic length scales, namely, the initiation length and the ODW surface position, are defined and discussed. When $\mathrm{ER}_{\mathrm{b}}$ increases, the initiation length curve has three trends, i.e., rapid decrease, modest decrease and modest increase. Nevertheless, the ODW surface position defined here is insensitive to the ER variation, different from the initiation length. By perform- 
ing further computations using the reversed ER distribution, their dependence on the ER inhomogeneity is discussed, demonstrating the initiation length is mainly determined by the ER of the lower portion of the incoming flow before the oblique shock, while the ODW position is significantly affected by the upper ER variation before the ODW surface.

The effects of ER inhomogeneity have been investigated and the distorted wave structures, such as the V-shaped deflagration, are discussed in previous studies [34,38]. The structures observed in this study are different slightly from those previous ones, primarily due to the different inhomogeneity settings. The choice of linear ER inhomogeneity before the OSW represents actually the simplest situation, which may still be far from the realistic operating conditions of practical ODW engines. However, this ideal study reveals how the inhomogeneity of the initiation region influences the wave structure and characteristic parameters, and builds up the connection between the structures in homogeneous and inhomogeneity mixtures. This can be viewed as the preliminary step towards a more quantitative and realistic study. Finally the collective effects of various inhomogeneity distributions need to be synthesized as the basis of application, and further study on the effects of inhomogeneity before the ODW surface is necessary.

\section{Conflict of interest statement}

No conflict of interest.

\section{Acknowledgement}

The research is supported by The National Natural Science Foundation of China NSFC Nos. 91641130 and 11372333.

\section{References}

[1] K. Kailasanath, Recent developments in the research on pulse detonation engines, AIAA J. 41 (2003) 145-159.

[2] A.J. Higgins, Ram accelerators: outstanding issues and new directions, J. Propuls. Power 22 (2006) 1170-1187.

[3] P. Wokanski, Detonative propulsion, Proc. Combust. Inst. 34 (1) (2013) $125-158$.

[4] E.M. Braun, F.K. Lu, D.R. Wilson, J.A. Camberos, Airbreathing rotating detonation wave engine cycle analysis, Aerosp. Sci. Technol. 27 (2013) 201-208.

[5] E.P. Gross, Hydrodynamics of a superfluid condensate, J. Math. Phys. 4 (2) (1963) 195-207.

[6] D.T. Pratt, J.W. Humphrey, D.E. Glenn, Morphology of standing oblique detonation waves, J. Propuls. Power 7 (5) (1991) 837-845.

[7] S.A. Ashford, G. Emanuel, Wave angle for oblique detonation waves, Shock Waves 3 (4) (1994) 327-329.

[8] G. Emanuel, D.G. Tuckness, Steady, oblique, detonation waves, Shock Waves 13 (6) (2004) 445-451

[9] C. Li, K. Kailasanath, E.S. Oran, Detonation structures behind oblique shocks, Phys. Fluids 6 (4) (1994) 1600-1611.

[10] L.F. Figueira Da Silva, B. Deshaies, Stabilization of an oblique detonation wave by a wedge: a parametric numerical study, Combust. Flame 121 (1) (2000) 152-166.

[11] M.V. Papalexandris, A numerical study of wedge-induced detonations, Combust. Flame 120 (4) (2000) 526-538.

[12] H.H. Teng, Z.L. Jiang, On the transition pattern of the oblique detonation structure, J. Fluid Mech. 713 (2012) 659-669.

[13] J.Y. Choi, E.J.R. Shin, I.S. Jeung, Unstable combustion induced by oblique shock waves at the non-attaching condition of the oblique detonation wave, Proc. Combust. Inst. 32 (2) (2009) 2387-2396.

[14] H.H. Teng, Z.L. Jiang, H.D. Ng, Numerical study on unstable surfaces of oblique detonations, J. Fluid Mech. 744 (2014) 111-128.

[15] Y. Liu, D. Wu, S. Yao, J. Wang, Analytical and numerical investigations of wedgeinduced oblique detonation waves at low inflow Mach number, Combust. Sci Technol. 187 (6) (2015) 843-856.

[16] Y. Liu, X. Han, S. Yao, J. Wang, A numerical investigation of the prompt oblique detonation wave sustained by a finite-length wedge, Shock Waves 26 (6) (2016) 729-739
[17] S. Bhattrai, H. Tang, Formation of near-Chapman-Jouguet oblique detonation wave over a dual-angle ramp, Aerosp. Sci. Technol. 63 (2017) 1-8.

[18] M.J. Grismer, J.M. Powers, Numerical predictions of oblique detonation stability boundaries, Shock Waves 6 (3) (1996) 147-156.

[19] M.Y. Gui, B.C. Fan, G. Dong, Periodic oscillation and fine structure of wedgeinduced oblique detonation waves, Acta Mech. Sin. 27 (6) (2011) 922-928.

[20] J.Y. Choi, D.W. Kim, I.S. Jeung, F. Ma, V. Yang, Cell-like structure of unstable oblique detonation wave from high-resolution numerical simulation, Proc. Combust. Inst. 31 (2) (2007) 2473-2480.

[21] J. Verreault, A.J. Higgins, R.A. Stowe, Formation of transverse waves in oblique detonations, Proc. Combust. Inst. 34 (2) (2013) 1913-1920.

[22] H. Teng, H.D. Ng, K. Li, C. Luo, Z. Jiang, Evolution of cellular structures on oblique detonation surfaces, Combust. Flame 162 (2) (2015) 470-477.

[23] D.R. Wilson, F.K. Lu, H. Kim, R. Munipalli, Analysis of a Pulsed Normal Detonation Wave Engine Concept, AIAA paper 2001-1784, 2001.

[24] R. Munipalli, V. Shankar, D.R. Wilson, H. Kim, F.K. Lu, P.E. Hagseth, A Pulse Detonation Based Multimode Engine Concept, AIAA paper 2001-1786, 2001.

[25] F.K. Lu, H.Y. Fan, D.R. Wilson, Detonation waves induced by a confined wedge, Aerosp. Sci. Technol. 10 (2006) 679-685.

[26] H.Y. Fan, F.K. Lu, Numerical modelling of oblique shock and detonation waves induced in a wedged channel, Proc. Inst. Mech. Eng., G J. Aerosp. Eng. 222 (2008) 687-703.

[27] J.L. Cambier, H. Adelman, G.P. Menees, Numerical simulations of an oblique detonation wave engine, J. Propuls. Power 6 (3) (1990) 315-323.

[28] V.V. Vlasenko, V.A. Sabel'nikov, Numerical simulation of inviscid flows with hydrogen combustion behind shock waves and in detonation waves, Combust. Explos. Shock Waves 31 (3) (1995) 376-389.

[29] G. Fusina, J.P. Sislian, B. Parent, Formation and stability of near ChapmanJouguet oblique detonation waves, AIAA J. 43 (7) (2005) 1591-1604.

[30] B. Zhang, H.D. Ng, J.H.S. Lee, The critical tube diameter and critical energy for direct initiation of detonation in $\mathrm{C}_{2} \mathrm{H}_{2} / \mathrm{N}_{2} \mathrm{O} / \mathrm{Ar}$ mixtures, Combust. Flame 159 (9) (2012) 2944-2953.

[31] B. Zhang, N. Mehrjoo, H.D. Ng, et al., On the dynamic detonation parameters in acetylene-oxygen mixtures with varying amount of argon dilution, Combust. Flame 161 (5) (2014) 1390-1397.

[32] B. Zhang, C.H. Bai, Methods to predict the critical energy of direct detonation initiation in gaseous hydrocarbon fuels - an overview, Fuel 117 (2014) 294-308.

[33] B. Zhang, The influence of wall roughness on detonation limits in hydrogenoxygen mixture, Combust. Flame 169 (2016) 333-339.

[34] J.P. Sislian, R. Dudebout, J. Schumacher, M. Islam, T. Redford, Incomplete mixing and off-design effects of shock-induced combustion ramjet performance, J. Propuls. Power 16 (2000) 41-48.

[35] Y. Zhang, J. Gong, T. Wang, Numerical study on initiation of oblique detonations in hydrogen-air mixtures with various equivalence ratios, Aerosp. Sci. Technol. 49 (2016) 130-134

[36] J.H.S. Lee, The Detonation Phenomenon, Cambridge University Press, New York, 2008

[37] K. Iwata, S. Nakaya, M. Tsue, Numerical investigation of the effects of nonuniform premixing on shock-induced combustion, AIAA J. 54 (5) (2016) $1682-1692$.

[38] K. Iwata, S. Nakaya, M. Tsue, Wedge-stabilized oblique detonation in an inhomogeneous hydrogen-air mixture, Proc. Combust. Inst. 36 (2) (2017) $2761-2769$

[39] T. Wang, Y. Zhang, H. Teng, Z. Jiang, H. Ng, Numerical study of oblique detonation wave initiation in a stoichiometric hydrogen-air mixture, Phys. Fluids 27 (2015) 096101

[40] C. Li, K. Kailasanath, E.S. Oran, Effects of Boundary Layers on Oblique Detonation Structures, AIAA paper 93-0450, 1993.

[41] B.J. McBride, M.J. Zehe, S. Gordon, NASA Glenn Coefficients for Calculating Thermodynamic Properties of Individual Species, Report No. 2002-211556, NASA/TP, 2002

[42] M. Sun, K. Takayama, Conservative smoothing on an adaptive quadrilateral grid, J. Comput. Phys. 150 (1999) 143-180.

[43] E.F. Toro, Riemann Solvers and Numerical Methods for Fluid Dynamics, second ed., Springer, Berlin, 1999.

[44] R.J. Kee, F.M. Rupley, E. Meeks, J.A. Miller, Chemkin-II: a Fortran chemical kinetics package for the analysis of gas-phase chemical and plasma kinetics, Sandia National Laboratories, UC-405, SAND96-8216, 1989.

[45] P.N. Brown, G.D. Byrne, A.C. Hindmarsh, VODE, a variable-coefficient ODE solver, SIAM J. Sci. Stat. Comput. 10 (1989) 1038-1051

[46] R. Dudebout, J.P. Sislian, R. Oppitz, Numerical simulation of hypersonic shockinduced combustion ramjets, J. Propuls. Power 14 (1998) 869-879.

[47] D.C. Alexander, J.P. Sislian, B. Parent, Hypervelocity fuel/air mixing in mixedcompression inlets of shcramjets, AIAA J. 44 (2006) 2145-2155.

[48] H. Teng, Y. Zhang, Z. Jiang, Numerical investigation on the induction zone structure of the oblique detonation waves, Comput. Fluids 95 (2014) 127-131. 